\title{
Metodología de inspección higrotérmica para la determinación de un factor intensi- dad de evaporación en edificios históricos
}

\section{Hygrothermal inspection to determine the "evaporation intensity factor (Fi)" in heritage buildings}

$\underline{\text { S. García-Morales }}{ }^{(*)}$, L. López-González ${ }^{(*)}$, A. Collado-Gómez ${ }^{(*)}$

RESUMEN

En este artículo se presenta lo que hemos denominado "inspección higrotérmica", que consiste en un primer paso en el diagnóstico de flujos de agua a través de los elementos constructivos. El fundamento se basa en que un muro afectado por un problema de capilaridad está sometido a una succión con un determinado caudal y esta agua evaporará en la superficie del muro o del suelo, incrementando el contenido en humedad (W) de la lámina de aire en contacto con el paramento.

Se describe el método de inspección y de análisis de los resultados. Se utiliza un termohigrómetro de lectura directa, y las lecturas se hacen siguiendo un protocolo que permite el análisis de variables.

Los niveles de intensidad de los focos se establecen a partir de lo que hemos denominado "Factor de Intensidad de evaporación (Fi)". Se ilustra el método con ejemplos de estudios en varios edificios históricos

\section{SUMMARY}

This paper presents what we call "Hygrothermal Inspection". It consists in a first step in the way to quantify real water flux across constructions. Theoretical aspects are related to the fact that a wall, affected by rising damp, suctions water that will then evaporate from the surface, towards the internal and external air. This flux increases the humidity ratio of the internal air, mainly in the thin air coat in contact with the wall or pavement. This fact allows us to measure the relative evaporation intensity by means of a thermohygrometer and to map evaporation zones.

Measuring method and results analysis are shown. Different evaporation levels from the walls or pavements are established by means of calculating what we call "Evaporation Intensity Factor (Fi)". Some results from several years work on Heritage Buildings are presented.
Palabras clave: Humedades; diagnóstico; termohigrómetro; evaporación; ensayos no destructivos (NDT); ensayos in situ; edificios históricos.
Keywords: Dampness; diagnosis; non-destructive testing (NDT) : termohygrometer; evaporation; "in situ" testing; heritage buildings. 


\section{ANTECEDENTES}

Una de las áreas prioritarias de estudios sobre el Patrimonio está relacionada con la patología de humedades, y, entre las líneas de investigación sobre esta materia, una se refiere al desarrollo de ensayos no destructivos (non-destructive testings, (NDT)), otros "minimamente destructivos" (minordamage testings (MDT)) y los ensayos de monitorización (monitoring) aplicados a estructuras y edificios, principalmente de fábrica, que constituyen el grupo de sistemas constructivos más tradicionales. Los ensayos de monitorización, generalmente in-situ, se configuran como estudios intermedios entre el laboratorio y los proyectos de Intervención, y son ampliamente acogidos porque aportan información sobre situaciones reales, permitiendo establecer diseños experimentales con variables reales, no sólo en condiciones de laboratorio. Son indispensables para el análisis y diagnóstico de edificios ya construidos (1).

El caso concreto del diagnóstico de humedades en edificios históricos no cuenta todavía con una herramienta metodológica completa que, desde la sintomatología y mediante instrumentación, acceda a la causa de las lesiones. Normalmente, las fases de un diagnóstico son:

1. Inspección visual y análisis de la documentación

2. Hipótesis o prediagnóstico

3. Ensayos de comprobación

La inspección visual se apoya en la observación de las lesiones existentes en el edificio y sus materiales. Para el diagnóstico de humedades son sintomáticas las manchas, eflorescencias, el crecimiento biológico, el deterioro de los materiales, etc. De todos ellos, el más relevante es el estudio de las manchas, porque los datos sobre morfología, modo de aparición, situación, etc, permiten con frecuencia el planteamiento de las posibles hipótesis. Para muchas lesiones, sobre todo cuando son recientes, es posible establecer una relación causa-efecto que conduzca al diagnóstico.

Si embargo, en muchas ocasiones las lesiones que aparecen en edificios históricos "engañan", no son coherentes en su apariencia actual con las causas que las produjeron inicialmente. La presencia de sales higroscópicas, o de pátinas biológicas o de suciedad interfiere en el diagnóstico (2). Podría ocurrir, por ejemplo, que grandes manchas sean tan solo residuales, o fruto de condensación higroscópica, y los muros no estén realmente evaporando. En otras ocasiones ocurre lo contrario: un muro en aparente buen estado está sometido a una humedad que evapora a través de su superficie, sin que aparezca mancha visible en el paramento. En estos casos se necesita una estrategia complementaria para el planteamiento de las hipótesis de prediagnóstico; éste es el papel de la inspección higrotérmica que proponemos.

Los primeros intentos de sistematización científica, proponiendo la toma de datos mediante instrumentación, y no sólo la inspección visual, se deben ya a Giovanni Massari (3) a finales de los años 50, quien propuso por primera vez la utilización de termohigrómetros para la medida de las condiciones de humedad y temperatura del aire en los edificios a estudiar, si bien la utilidad de estos estudios sólo se confirmaba en problemas de condensación, y no en humedades del terreno o de filtración. Tampoco proponía Massari ningún método instrumental para la medida de la humedad en los materiales que componían muros o suelos, pues la tecnología no los había desarrollado. Para cuantificar la humedad presente en los materiales no se disponía entonces de otra técnica que la gravimétrica, (que consiste en tomar muestras de los materiales, Ilevarlos al laboratorio, y secarlos, para establecer así el porcentaje de humedad perdido en el secado), o técnicas similares como la del carburo de calcio. Estos ensayos son de tipo MDT (mínimamente destructivos, aunque las muestras pueden ser pequeñas), y por lo tanto su utilización es limitada.

En la práctica, todavía la bibliografía sobre humedades se limita a la descripción de síntomas asociados a distintos tipos de humedad, y la base del diagnóstico se basa más en la experiencia que en ninguna herramienta diagnóstica (4), (5). En Inglaterra, el BRE propugna desde hace años los mismos métodos para el diagnóstico de humedades, e incluso cuando se hacen mediciones sobre contenidos en humedad de los materiales o del ambiente, es difícil asociar estas mediciones con un diagnóstico concluyente. La línea de trabajo, para muchos, se centra todavía en encontrar herramientas de representación para la cartografía de lesiones (6).

La investigación sobre humedades en los edificios ha seguido dos líneas diferentes:

- Una de ellas hace, desde hace años, estudios de monitorización de los edificios (7), mediante la instalación de termohigrómetros, para analizar la evolución de temperaturas y humedad del ambiente interior, en relación a las variaciones higrotérmicas del aire exterior. Se trata de una línea ampliamente desarrollada como ayuda en la conservación de bienes muebles, colec- 
ciones, control ambiental museístico, etc. Se utilizan termohigrómetros de tipo datalogger de registro automático. El avance en este campo viene ligado al desarrollo de instrumentación inalámbrica (8).

- La otra línea de investigación se centra en el desarrollo de sensores para la medida del contenido en humedad de los materiales "in situ". Esta investigación camina hacia la invención de nuevos tipos de sensores y técnicas, si bien todavía no se ha llegado a la precisión que proporcionan los métodos gravimétricos. Actualmente, para la determinación del contenido en humedad de los muros de fábrica, los métodos existentes son (9):

\section{Metodos indirectos:}

- Termografía infrarroja, mediante sensores puntuales o mediante cámara IR. La Termografía infrarroja se viene utilizando desde el año 78, pero aún debe mejorarse el método, porque las medidas obtenidas a veces sólo se refieren a contenidos en humedad superficiales, y en otras ocasiones, la influencia del aire húmedo del interior de los edificios analizados distorsiona las mediciones o las imposibilita, aunque tiene las indiscutibles ventajas de rapidez y posibilidad de fotografiar el estado conjunto del paramento observado (10).

- Métodos basados en la medida de propiedades eléctricas. La medida de las propiedades eléctricas de los materiales es un medio para determinar su contenido en humedad. Inicialmente se desarroIlaron métodos apoyados en la medida de la resistencia eléctrica, con resultados mediocres. Se han ensayado con más éxito métodos apoyados en la medida de la capacitancia o impedancia, que tienen mayor penetración, aunque también se ven interferidos por factores superficiales, que distorsionan las lecturas. Al final, las técnicas eléctricas terminan siendo, cuando se utilizan en campo, más prospectivas que cuantitativas, si bien en laboratorio llegan a proporcionar valores bastante precisos (11).

- Medida de la humedad relativa en perforaciones abiertas en el muro, de acuerdo a normas de ensayo como la BS 82101 y la BS 53252. Este sistema se basa en la apertura de agujeros en las fábricas, en las que se introducen sondas de temperatura y humedad ambiental. Se trata de establecer correlaciones entre estos parámetros y el contenido en humedad de los materiales, pero los resultados no tienen valor nada más que en el caso de muros en los que el contenido en humedad es ya bajo, no cuando se trata de muros muy mojados.

\section{Metodos directos:}

- Son los más tradicionales, y todavía no han sido aventajados por las técnicas anteriores. Básicamente siguen siendo métodos gravimétricos aplicados a muestras de material extraídas del edificio (ensayos considerados de tipo MDT, porque las muestras extraídas son pequeñas, o incluso polvo) (12). Una mejora interesante de este método es la publicada por Sandrolini y otros (9).

\section{OBJETIVOS DE LA PRESENTE INVESTIGACIÓN}

Las dos líneas de investigación descritas más arriba no confluyen para aportar una herramienta diagnóstica útil en los problemas de humedad en edificios históricos. Por ello, desde 1992 desarrollamos una metodología nueva en este sentido (13), que combinaba la toma de datos instrumental con una variación del método gravimétrico. La metodología se demostraba útil y con su ayuda se pudieron diagnosticar las humedades de numerosos edificios del $\mathrm{Pa}$ trimonio, algunos publicados (14).

Al continuar con el desarrollo de esta metodología en estos años, hemos tratado de orientar la toma de datos analizando los flujos de agua a través de los elementos constructivos, más que incidir en los contenidos en humedad de los materiales en un momento determinado. Esto significa, por ejemplo, que un muro afectado por un problema de capilaridad (Figura 1) está sometido a una succión con un determinado caudal (por ejemplo, en kg de agua por metro lineal de zapata de cimentación), y que esta agua ascenderá por el muro y se evaporará en el zócalo, de tal manera que, cuando se observa el zócalo húmedo de un edificio, aunque la apariencia de la lesión sea que el agua "está", la realidad es que el agua "fluye". El agua evapora en la superficie del muro o del suelo, y pasa al ambiente (interior y exterior). Esta humedad-vapor aumenta el contenido en humedad del aire interior (sobre todo la humedad de la lámina de aire en contacto directo con el paramento), y es precisamente este hecho lo que fundamenta el presente método de trabajo, que busca cuantificar de alguna manera la intensidad del foco de evaporación que se produce desde el muro o solera húmedos, presentando un método nuevo y sencillo.

En este artículo se presenta lo que hemos denominado "inspección higrotérmica", cuya finalidad es cartografiar y cuantificar
1. Esquema de la evaporación en un muro sometido a humedades de capilaridad.

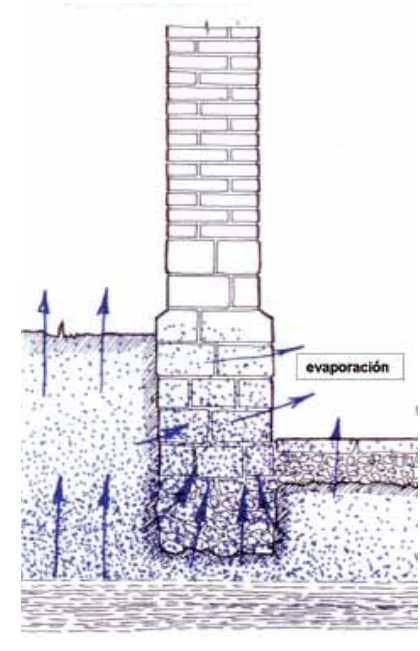

1 
2. Termohigrómetro utilizado en la inspección higrotérmica.

3. Ejemplo de utilización del termohigrómetro en la inspección.

4. Esquema de toma de datos según variables. la intensidad relativa de los focos de evaporación en un edificio, clasificándolos según niveles. Los niveles se establecen a partir de lo que hemos denominado "Factor de Intensidad de evaporación", como se describe más abajo. De esta forma, la mayor intensidad de un foco de evaporación detectado, se convierte en una variable que permite, en conjunción con otras, un prediagnóstico de las humedades.

\section{MÉTODO DE INSPECCIÓN}

La inspección higrotérmica consiste en la medida de temperatura $\left(\mathrm{T}\right.$, en $\left.{ }^{\circ} \mathrm{C}\right)$ y grado de humedad (W, expresado en gramos $/ \mathrm{kg}$ ) del aire en contacto con los paramentos de muros y soleras (El grado de humedad es una medida absoluta del contenido en humedad del aire, y se expresa en gramos de agua por $\mathrm{kg}$ de aire). La toma de datos se hace manualmente, en cada punto, con ayuda de un termohigrómetro de lectura instantánea (Figura 2). En los casos que se presentan en el presente trabajo se ha utilizado un termohigrómetro de lectura digital, marca TESTO 610 que mide temperatura, humedad relativa y punto de rocío del aire, y cuyos datos técnicos son (Tabla 1):

\begin{tabular}{|c|c|}
\hline & Tabla 1 \\
\hline \multicolumn{2}{|c|}{ Tipo de sonda: NTC } \\
\hline Exactitud & $\pm 0,5^{\circ} \mathrm{C}$ \\
\hline Resolución & $0,1^{\circ} \mathrm{C}$ \\
\hline \multicolumn{2}{|c|}{ Tipo de sonda Sensor de humedad, capacitivc } \\
\hline Exactitud & $\pm 2.5 \% \mathrm{HR}(+5 \ldots+95 \% \mathrm{HR})$ \\
\hline Resolución & $0,1 \% \mathrm{HR}$ \\
\hline
\end{tabular}

Mediante una tabla de cálculo se obtiene el valor del grado de humedad correspondiente a cada punto.

La lectura se obtiene apoyando la sonda sobre el punto del paramento sobre el que se desea tomar la lectura (Figura 3). Se espera el tiempo suficiente para que la lectura se estabilice, y en ese momento se anota el valor de temperatura y grado de humedad del aire en contacto con el paramento en ese punto.

\subsection{Protocolo de toma de datos}

Los datos se toman siguiendo una metodología científica, es decir, responden a una serie de hipótesis previas, que determinan un modo concreto de tomar las lecturas, clasificándolas de modo que permitan luego un análisis que pueda ser estadístico. Algunas de estas hipótesis de partida (por otra parte, bastante evidentes) son:

- En el interior de un edificio húmedo la humedad es mayor que en el exterior.

- Cuanto mayor es la humedad de una sala o ambiente interior, más cercano está el foco que lo produce
- Cuando en una sala el aire directamente en contacto con un muro o pavimento está más húmedo que el de la propia sala, ese muro o pavimento está evaporando.

- Cuanto más cerca está un foco de evaporación, mayor es la humedad del aire.

De este modo, la experiencia nos ha conducido a establecer unas variables de observación que se ajusten a estas hipótesis, y que son las siguientes:

- Variable "ambiente" (A): se establece en dos niveles de observación: interior y exterior.

- Variable "altura" (B): que se anota en cm. sobre la cota del suelo.

- Variable "posición" (C): en cuatro niveles (Figura 4):

- Punto situado en el centro de una sala o recinto (lecturas designadas como tipo "a")

- Punto situado sobre la solera (lecturas designadas como " $\mathrm{s}$ ")

- Punto situado en la arista entre suelo y pared, (o entre suelo y pilar): lecturas designadas como " $\mathrm{sp}^{\prime \prime}$ )

- Punto situado en pared (lecturas designadas como " $p$ ")

- Variable "material" (D): que se refiere al material sobre el que se toma la lectura: mortero, ladrillo, piedra, o "hueco", en el caso de que se trate de agujeros en alguno de los materiales.

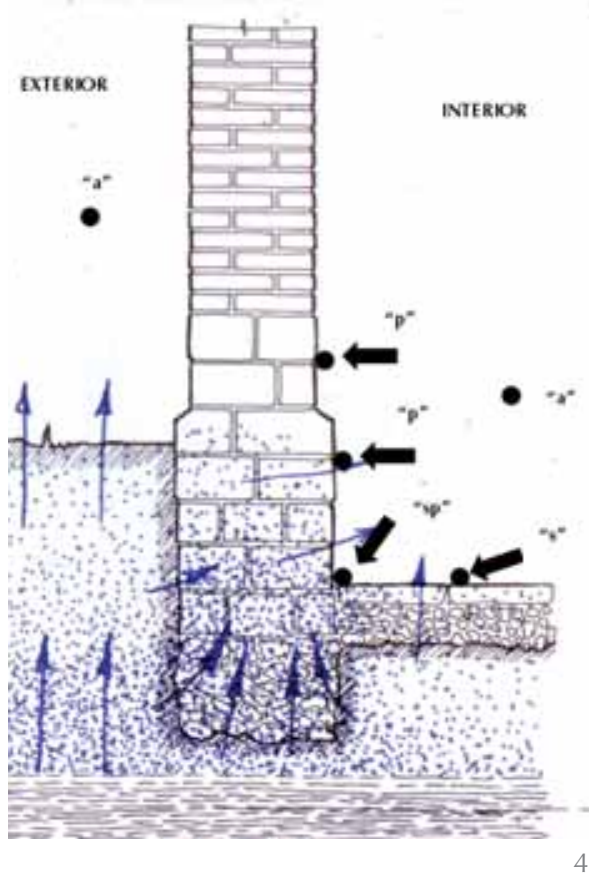

A estas variables observadas se pueden añadir otras, según la naturaleza de la hipótesis previa a la inspección. Podrían analizarse por separado diferentes salas, o muros según su orientación, por ejemplo, si se sospechara que esta variable puede influir en la humedad del edificio. 
Para la investigación se parte inicialmente del conocimiento del aire exterior, que constituye el punto de referencia para las lecturas interiores. Se toman un número suficiente de lecturas de ambiente exteriores (lecturas tipo "a"), a una altura de 1 metro sobre el suelo.

A continuación se toman lecturas tipo "a" del aire interior, también a una altura de 1 metro sobre el suelo: el número de lecturas depende del tamaño del edificio. Al menos se toma una lectura en cada uno de los locales o salas que configuran el conjunto. En el presente artículo se comparan ejemplos de inspección higrotérmica en iglesias, en las que el ambiente interior es bastante único, al carecer de compartimentación.

Cuando se desea analizar la ventilación o la posible estratificación del aire húmedo, se toman lecturas tipo "a" a diferentes alturas, no sólo a 1 metro sobre el suelo. En ocasiones se ha aprovechado la instalación existente de un andamio, o una escalera de acceso a los triforios, para tomar este tipo de medidas.

Cuando se tiene un número suficiente de lecturas tipo "a" como para poder caracterizar el ambiente interior, se procede a la toma de datos de tipo "s", "sp" y "p".

Las lecturas de suelo (" $\mathrm{s}$ ") se obtienen apoyando la sonda directamente sobre el pavimento. Las lecturas " $\mathrm{sp}^{\text {" se obtienen }}$ apoyando la sonda en la línea que marca el encuentro entre el pavimento y el muro (o sobre el remate superior del zócalo, en el caso de que exista). Para las lecturas tipo " $\mathrm{p}$ " se pone en contacto la sonda con el paramento vertical.

El número de lecturas depende del tipo de edificio a estudiar, y de la magnitud de los focos detectados. Es importante la velocidad de respuesta del termohigrómetro, que es, en definitiva, lo que determina el número de lecturas que se pueden tomar en un día. En las inspecciones realizadas, se ha tomado una media de 100 lecturas en una mañana de trabajo.

En la práctica, la precisión del método de inspección aquí presentado oscila en torno a $0,5 \mathrm{~g} / \mathrm{kg}$. Esto significa que no son significativas diferencias de valor menores de este valor.

\subsection{Método de análisis de resultados}

Las lecturas se anotan inicialmente en tablas similares a esta: (Tabla 2)
Una vez tomados los datos, se realiza un análisis estadístico de los resultados, según las variables estudiadas. En dicho análisis se considera como "umbral de referencia" el aire exterior, que en principio debería presentar valores mínimos de grado de humedad.

Si se hacen inspecciones en el mismo edificio, en días distintos, la temperatura y grado de humedad exteriores varían, lógicamente. Por ello las lecturas tomadas en el interior siempre se comparan con los valores del "aire de referencia" exterior del día correspondiente a la inspección.

Los resultados se analizan mediante gráficos. En los gráficos de comparación temperatura/grado de humedad se obtiene una nube de puntos cuyos valores máximos ( $\mathrm{W}_{\text {i }}$ ) son indicativos de la intensidad general de la evaporación medida en el edificio. De este modo, representamos el "Factor Intensidad Máximo de evaporación ( $\mathrm{Fi}{ }_{\max }$ )" mediante esa diferencia, que no es más que la "altura" de la nube de puntos con respecto al grado de humedad del aire de referencia, es decir: [1]

[1] $\quad \mathrm{Fi}_{\max }=\mathrm{W}_{\mathrm{i} \max }-\mathrm{W}_{\mathrm{e}}($ en gramos $/ \mathrm{kg})$

Para una mejor interpretación de los datos, se hace una cartografía de los focos, con indicación de "niveles de intensidad" sobre planos del edificio, lo que permite avanzar el prediagnóstico, de la siguiente forma:

- Cuando se detectan focos de evaporación continuos a lo largo de un muro, y estos son más significativos en puntos "sp", el prediagnóstico señala una posible humedad desde el terreno.

- Cuando se trata de muros en los que se sospecha filtración de agua de lluvia desde el exterior, es preciso comprobar tanto en los puntos " $\mathrm{sp}^{\mathrm{p}}$ como en puntos " $\mathrm{p}$ ", para detectar si la evaporación es más intensa desde cotas superiores o desde la cota del pavimento. La correlación de las alturas en las que se miden focos más intensos puede informar sobre las áreas de filtración (zócalo, acera, embolsamiento a pie de muro...)

- Cuando se detectan focos de evaporación más intensos en el pavimento (puntos "s") que en los encuentros con los muros (puntos " $\mathrm{sp}^{\prime \prime)}$, el prediagnóstico orienta hacia humedades internas del edificio, posiblemente procedentes de averías, pozos, etc.

Tabla 2

\begin{tabular}{|c|c|c|c|c|c|c|c|c|}
\hline \multirow{2}{*}{ Hora } & \multirow{2}{*}{$\mathbf{o}$} & \multirow{2}{*}{$\mathrm{T}\left({ }^{\circ} \mathbf{C}\right)$} & \multirow{2}{*}{$\mathrm{W}(\mathrm{g} / \mathbf{k g})$} & \multicolumn{4}{|c|}{ Variables } & \multirow{2}{*}{ Otras anotaciones } \\
\cline { 5 - 8 } & & & & $\mathrm{A}$ & $\mathbf{B}(\mathbf{c m})$ & $\mathrm{C}$ & $\mathrm{D}$ & \\
\hline $10: 00$ & 1 & 15,2 & 4,65 & int & 100 & "a" & ladrillo & \\
\hline & 2 & 15,4 & 6,28 & int & 1 & "sp" & mortero & \\
\hline & 3 & 15,3 & 4,75 & int & 20 & "p" & agujero & \\
\hline
\end{tabular}


6. Gráfico que representa las lecturas en San Miguel, de San Esteban de Gormaz.

5. Representación del Factor Intensidad de evaporación máximo (Fi max)y de la clasificación de las lecturas tomadas en dos iglesias de Zamora.

\section{EJEMPLO 1: INSPECCIÓN EN DOS IGLESIAS DE ZAMORA}

Puede verse un ejemplo en el gráfico (Figura 5) que representa los valores de temperatura $\mathrm{T}$ (representado sobre el eje $\mathrm{X}$ ) y grado de humedad W (eje Y) de la inspección higrotérmica realizada en dos iglesias distintas de Zamora, el mismo día 10 de julio de 2010. Una de ellas (Santa María la Nueva) se ha visitado por la mañana, y la otra (Santa María de Horta) por la tarde.

El grado de humedad exterior $\left(\mathrm{W}_{\mathrm{e}}=8,1 \mathrm{~g} /\right.$ $\mathrm{kg})$ se ha tomado como valor medio, pues varió ligeramente de la mañana a la tarde.

Lecturas en dos iglesias de zamora (10-VII-2010)

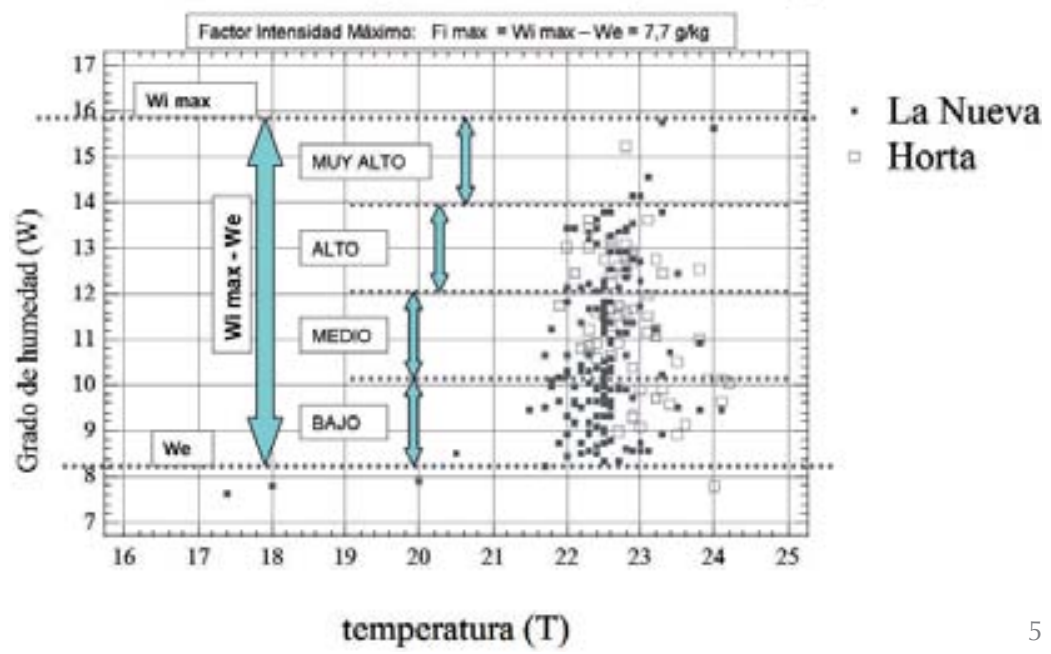

Inspección en la iglesia de San Miguel (San Esteban de Gormaz) (3-IV-2007)

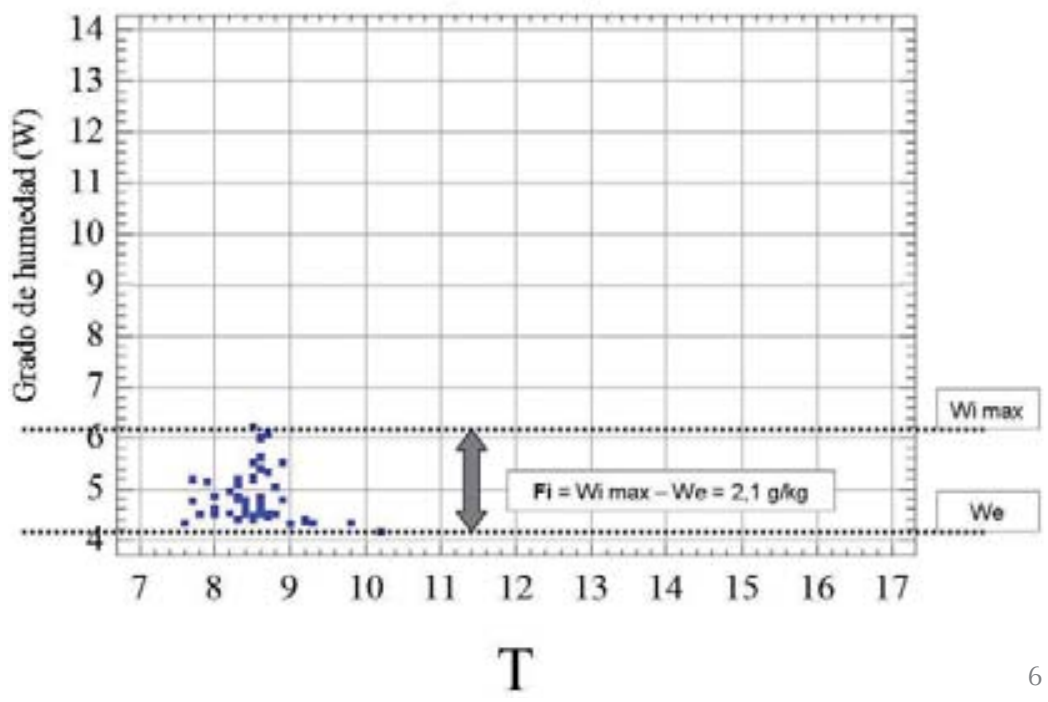

Tabla 3

\begin{tabular}{|l|l|}
\hline & Puntos en los que se cumple que... \\
\hline Foco de intensidad "bajo" & $0<\left(\mathrm{W}_{\mathrm{i}}-\mathrm{W}_{\mathrm{e}}\right)<\left(\mathrm{W}_{\mathrm{i}} \max -\mathrm{W}_{\mathrm{e}}\right) / 4$ \\
\hline Foco de intensidad "medio" & $\left(\mathrm{W}_{\mathrm{i}} \mathrm{max}-\mathrm{W}_{\mathrm{e}}\right) / 4<\left(\mathrm{W}_{\mathrm{i}}-\mathrm{W}_{\mathrm{e}}\right)<2\left(\mathrm{~W}_{\mathrm{i}} \max -\mathrm{W}_{\mathrm{e}}\right) / 4$ \\
\hline Foco de intensidad "alto" & $2\left(\mathrm{~W}_{\mathrm{i}} \mathrm{max}-\mathrm{W}_{\mathrm{e}}\right) / 4<\left(\mathrm{W}_{\mathrm{i}}-\mathrm{W}_{\mathrm{e}}\right)<3\left(\mathrm{~W}_{\mathrm{i}} \max -\mathrm{W}_{\mathrm{e}}\right) / 4$ \\
\hline Foco de intensidad "muy alto" & $3\left(\mathrm{~W}_{\mathrm{i}} \mathrm{max}-\mathrm{W}_{\mathrm{e}}\right) / 4<\left(\mathrm{W}_{\mathrm{i}}-\mathrm{W}_{\mathrm{e}}\right)<\left(\mathrm{W}_{\mathrm{i}} \max -\mathrm{W}_{\mathrm{e}}\right)$ \\
\hline
\end{tabular}

Se puede observar que las dos iglesias tienen una temperatura bastante constante a lo largo del día (entre 22 y 24 grados) y un Factor Intensidad Máximo $\left(\mathrm{Fi}_{\max }\right)$ bastante similar $(7,7 \mathrm{~g} / \mathrm{kg})$.

Para clasificar los focos de evaporación detectados, se divide la nube de puntos en cuatro niveles, estableciendo niveles del Fi en cada nivel, en este caso, según las siguientes expresiones: (Tabla 3 ).

La representación de los cuatro niveles se aprecia en el gráfico de la Figura 5.

Finalmente, se representan sobre planos del edificio los niveles de humedad detectados, y este plano es el que se utiliza como referencia para el prediagnóstico de la humedad.

\section{EJEMPLO 2: INSPECCIÓN EN LA IGLE- SIA DE SAN MIGUEL (SAN ESTEBAN DE GORMAZ, SORIA)}

Esta iglesia, de finales siglo XI, es la primera iglesia porticada del románico de Soria. Se trata de una iglesia de una nave con ábside semicircular, torre y atrio porticado. La inspección higrotérmica se presenta en el gráfico de la Figura 6. Durante el día de la inspección, el grado de humedad exterior $\left(\mathrm{W}_{\mathrm{e}}\right)$ era de 4,1 $\mathrm{g} / \mathrm{kg}$.

5 Es interesante comparar esta iglesia con la del ejemplo anterior, porque en este caso se puede apreciar que el factor $\mathrm{Fi}_{\text {max }}$ es aquí mucho más bajo: $(2,1 \mathrm{~g} / \mathrm{kg})$, lo cual indica que la iglesia presenta focos de evaporación más pequeños que en el caso anterior. Si se realiza la misma clasificación de las lecturas por "niveles" y se cartografían los puntos sobre la planta del edificio, se puede apreciar que en el plano aparecen marcados las zonas en las que la intensidad de evaporación es mayor (Figura 7). En este caso el diagnóstico es relativamente sencillo, pues ambos focos coinciden con zonas de riesgo de filtración desde el exterior, como se puede apreciar en las fotos (Figuras 8 y 9 ).

\section{EJEMPLO 3: INSPECCIÓN EN LA IGLESIA DE SAN PEDRO DE ÁVILA}

La iglesia de San Pedro de Ávila (Figura 10) comenzó a edificarse en torno al año 1100. Se clasifica dentro del estilo románico. La construcción debía estar completada hacia finales del XII. En el siglo XIV, debió ser rehecha debido a problemas de estabilidad estructural, y a lo largo del siglo $X X$ ha pasado por diversas intervenciones para solucionar problemas de humedad y de deterioro de la piedra. 


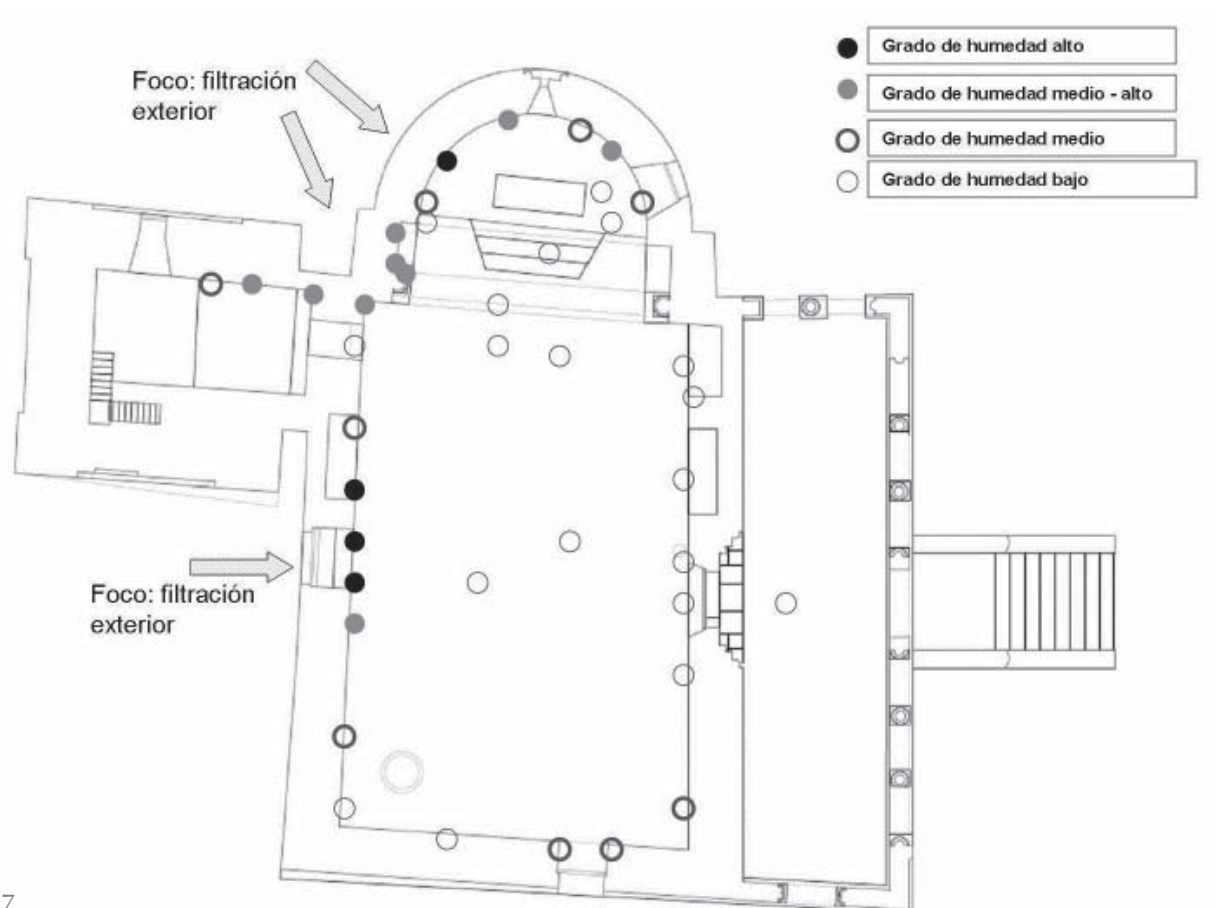

La inspección higrotérmica del edificio se llevó a cabo como primera fase dentro de un estudio global de diagnóstico de humedades, durante el año 2010. La toma de datos se realizó en dos días diferentes: el día 14 de abril y el 9 de julio de 2010, para tener datos de primavera y de verano (cuando se supone que el edificio puede evaporar con más eficacia, si se trata de humedades del terreno). Las lecturas se han realizado por la mañana. Los resultados se presentan en forma de gráficos, y para su interpretación se han cartografiado sobre planos de planta.

\subsection{Análisis de resultados de la inspección de San Pedro}

Se comparan (Figura 11) los valores de grado de humedad del aire $\left(\mathrm{W}_{\text {. }}\right.$ ) en los distintos puntos inspeccionados. Tal y como se ha indicado, en la inspección higrotérmica habría que tomar como referencia el valor del grado de humedad exterior $\left(\mathrm{W}_{\mathrm{e}}\right)$, pero en el día 14-IV, que era un día de Iluvia, este valor de grado de humedad exterior era mayor que algunos de las lecturas tomadas en el interior. Esta anomalía, que hemos observado en otras ocasiones cuando se hace la inspección en un día húmedo, la

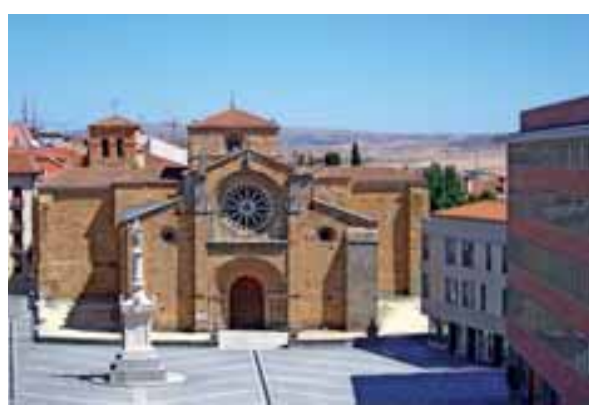

10 atribuimos al hecho de que en algunos edificios mal ventilados, el grado de humedad exterior varía con más rapidez que en el interior, y por eso, cuando se hace la inspección, el ambiente general del edificio no se corresponde con las condiciones exteriores del día en que se realiza el estudio. Cuando esto ocurre no sirve la referencia de aire exterior, y para establecer una, venimos tomando el valor mínimo entre los medidos en el interior del edificio $\left(W_{i \text { min }}\right)$. Esto no es arbitrario, si se recuerdan las nubes de puntos de las gráficas que venimos analizando, donde se aprecia que esto siempre es así. Por lo tanto, los valores de "aire de referencia" quedan como sigue:

- Para el día 14-IV: el exterior estaba en torno a $6 \mathrm{~g} / \mathrm{kg}$ pero, según lo que acabamos de comentar, se ha tomado 5,2 g/kg $\left(\mathrm{W}_{\mathrm{i} \text { min }}\right)$ como valor de referencia de aire seco exterior en este día.

Inspección en San Pedro de Avila

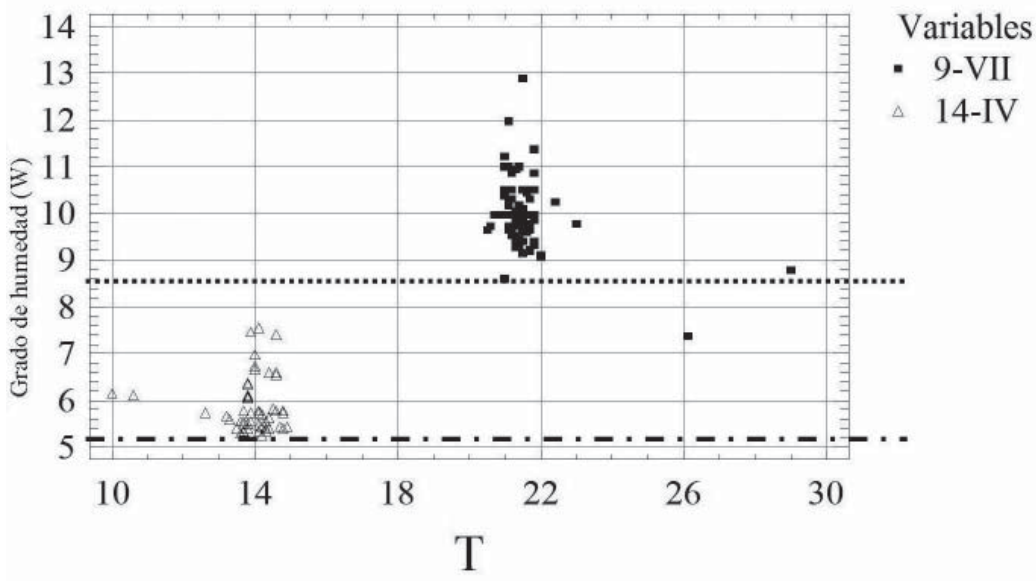


12. la misma nube de puntos, en la que se distinguen según la variable C.

13. Cartografía de los distintos niveles de los focos de evaporación en una parte de la cabecera de la iglesia de San Pedro de Ávila, el día 14 de abril de 2010.

14. Cartografía de 9 de Julio de 2010.
- El día 9-VII: W oscilaba alrededor de $8,5 \mathrm{~g} / \mathrm{kg}$.

En abril, el aire exterior contiene menos humedad que en verano, como es normal. La diferencia $\left(\mathrm{Fi}_{\max }=\mathrm{W}_{\mathrm{i} \max }-\mathrm{W}_{\mathrm{e}}\right.$ ) de la iglesia, sin embargo, no varía tanto de un mes a otro.

- En abril $\mathrm{Fi}_{\text {max }}=2,3 \mathrm{~g} / \mathrm{kg}$ aproximadamente, (para los puntos más desfavorables)

Inspección en San Pedro de Avila

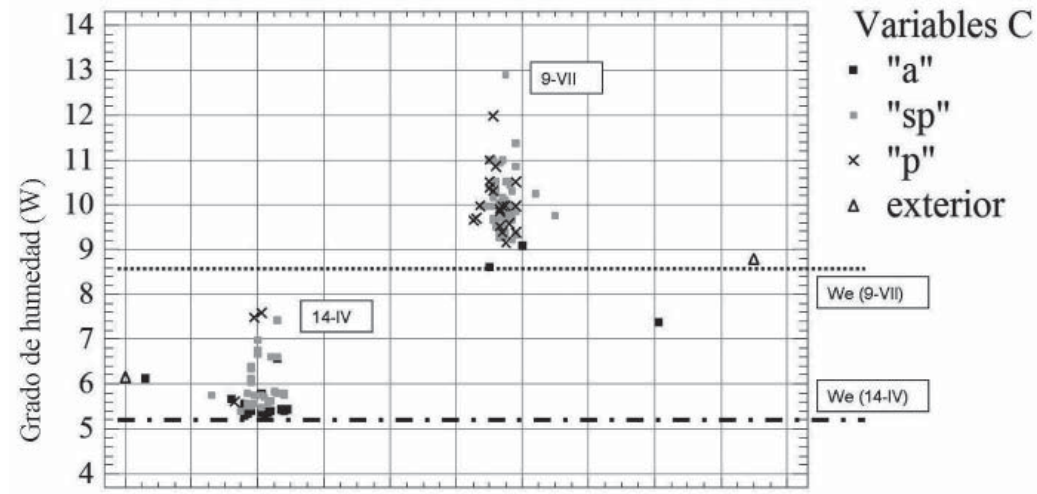

$\begin{array}{lllllllllll}10 & 12 & 14 & 16 & 18 & 20 & 22 & 24 & 26 & 28 & 30\end{array}$
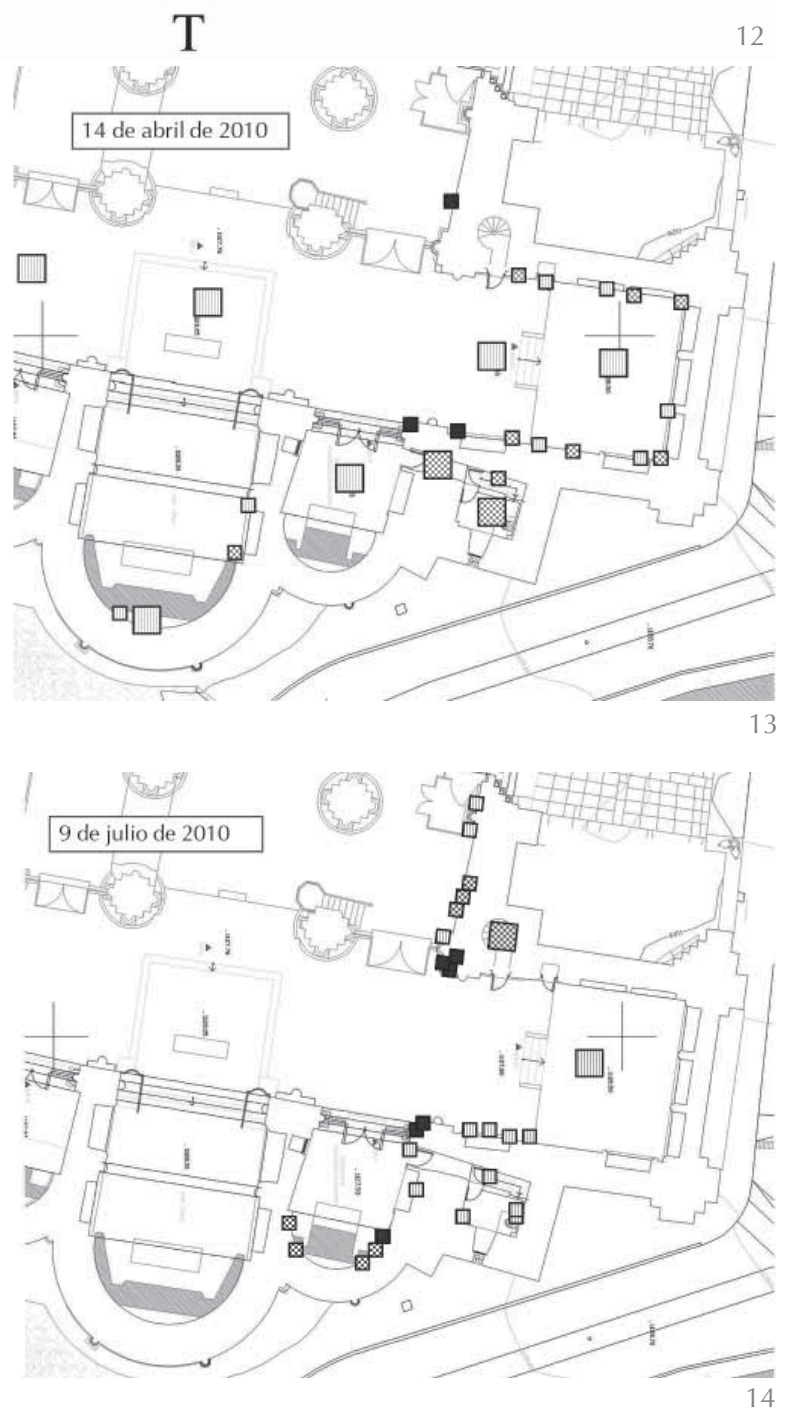

- En julio $\mathrm{Fi}_{\text {max }}=3,5 \mathrm{~g} / \mathrm{kg}$ aproximadamente (para los puntos más desfavorables)

Además, la mayoría de los valores medidos tienen poca diferencia con respecto a los valores de referencia. Esto significa que la evaporación, en el día de la inspección de abril, podría considerarse "baja", y en el mes de julio, de "media". Si se compara el valor de $\mathrm{Fi}_{\text {max }}$ de esta iglesia con los de las dos iglesias de Zamora inspeccionadas un día después (10-VII), la conclusión sería que la iglesia no está muy húmeda (evapora relativamente poco), tanto en abril como en julio.

Con relación a las temperaturas, la media en el día de abril era de $14{ }^{\circ} \mathrm{C}$, y en julio, de $21{ }^{\circ} \mathrm{C}$. El exterior estaba a $10^{\circ} \mathrm{C}$ y $22-28$ ${ }^{\circ} \mathrm{C}$, respectivamente.

Si distinguimos los distintos tipos de puntos en el gráfico según variables, se observa lo siguiente: (Figura 12).

Los valores de humedad "ambiente" son muy bajos en ambos casos. Los valores que se han tomado en el perímetro interior de la iglesia ("sp"), y en algunos puntos de la pared, son los realmente significativos, (aunque ya hemos indicado que son valores bajos o medios). Se han cartografiado algunos de estos puntos sobre una zona del plano de planta, para poder visualizarlos de un modo más gráfico (Figuras 13 y 14). Se puede observar cómo la posición de los focos y su intensidad relativa varía poco a pesar de que se tomen las medidas en días diferentes.

Como se puede ver en los dibujos, los focos de humedad son "dispersos", coincidiendo los más intensos con los cuatro pilares donde se encuentra el crucero y la nave, que coinciden, también, con los "rincones" del edificio, y que posiblemente posean las cimentaciones más masivas. Estos puntos se han podido después correlacionar con filtraciones de agua procedentes del vertido de pluviales a pie de muro, o acumuladas en rincones.

\section{EJEMPLO 4: INSPECCIÓN EN LA CATEDRAL DE GETAFE}

A continuación se presenta otro edificio en el que la evaporación es más intensa que en los casos anteriores. Se trata de la Catedral de Getafe (Figuras 15 y 16). La inspección se realizó en los días 5.VII y 26.VII.05. Se hicieron 102 medidas, tanto en la nave como en la sacristía. La inspección se centró en los zócalos, las paredes (hasta una altura de 2,0 metros), y el pavimento. La iglesia estaba en fase de excavación arqueológica y había bastante facilidad para la evaporación desde las catas abiertas en suelo y en pared. 
El grado de humedad de referencia exterior se tomó en $11 \mathrm{~g} / \mathrm{kg}$, por ser el valor máximo de los medidos alrededor de la Catedral.

El Factor Intensidad de evaporación $\mathrm{Fi}$ max era elevado (10,3 g/kg) (Figura 17), sobre todo en los puntos en los que había algún tipo de agujero abierto en el muro, o una grieta.

Gracias a la inspección se detectaron focos de evaporación (Figura 18) en los muros, sobre todo en los encuentros entre pilastras y muro, y también entre las jambas de las puertas y el muro. La humedad del muro se mide sobre todo en dos alturas (Figura 19):

- La base del muro

- La cota que coincide con el remate del zócalo del exterior.

\section{CONCLUSIONES}

1. La inspección higrotérmica puede ser una ayuda eficaz en el diagnóstico de humedades en edificios históricos, sobre todo en los casos en que la inspección de lesiones no es suficiente para aproximar un prediagnóstico.

2. Para que la toma de datos sea relevante, debe hacerse siguiendo una metodología científica. Se ha demostrado la utilidad de tomas las lecturas siguiendo un protocolo de inspección según variables, como se muestra en el trabajo, especialmente las variables " $\mathrm{C}$ " (posición del punto de lectura en ambiente, suelo, suelo/pared o pared), y la altura.

3. La inspección higrotérmica proporciona información sobre:

- La presencia de focos de evaporación significativos. Se considera significativo un foco de humedad en el que $W_{i \max }-W_{e}$ $>1 \mathrm{~g} / \mathrm{kg}$.

- La intensidad relativa de los focos de evaporación puede obtenerse a partir del Factor Intensidad de evaporación máxi$\operatorname{mo}\left(\mathrm{Fi}_{\max }=\mathrm{W}_{\mathrm{i} \max }-\mathrm{W}_{\mathrm{e}}\right)$, dividiéndolo en cuatro niveles, según se ha indicado en la Figura 5.

4. Cuando se realiza la inspección higrotérmica en días húmedos (lluvia o niebla), suele ocurrir que $W_{e}>W_{i}$ en muchos puntos. Se ha ilustrado este hecho en el ejemplo de abril en San Pedro. En estos casos, para la determinación de un $\mathrm{Fi}_{\text {max }}$ "corregido" que permita hacer una clasificación de los niveles de intensidad de los focos, se puede acudir a la expresión $F_{i \text { max }}=W_{i \text { max }}-W_{i \text { min }}$
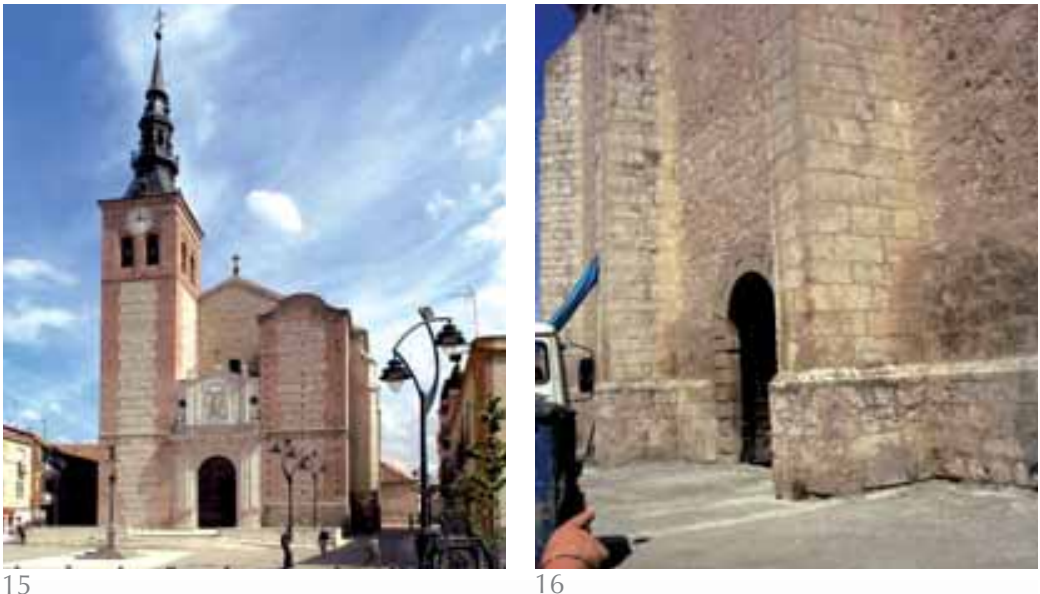

Inspección en la Catedral de Getafe

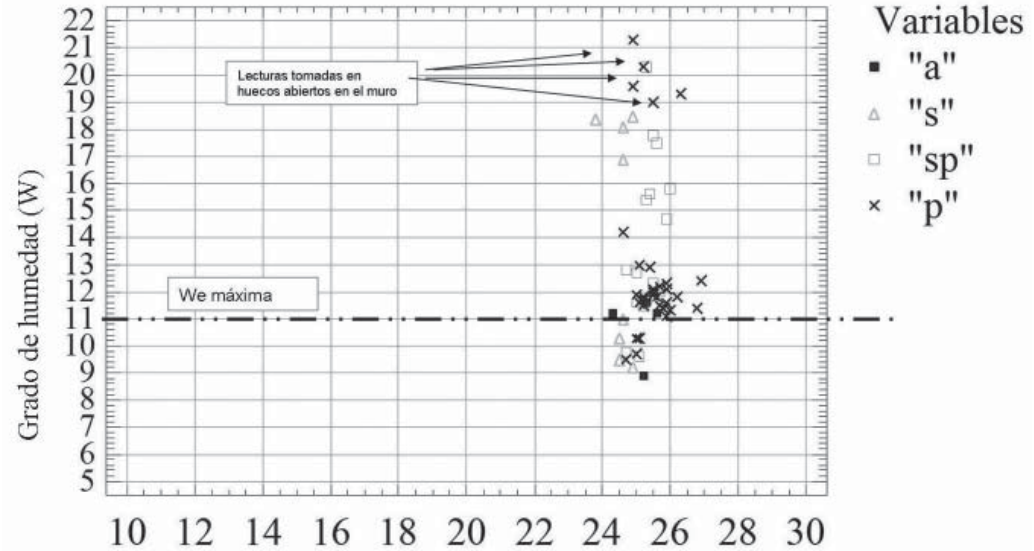

17

$\mathrm{T}$

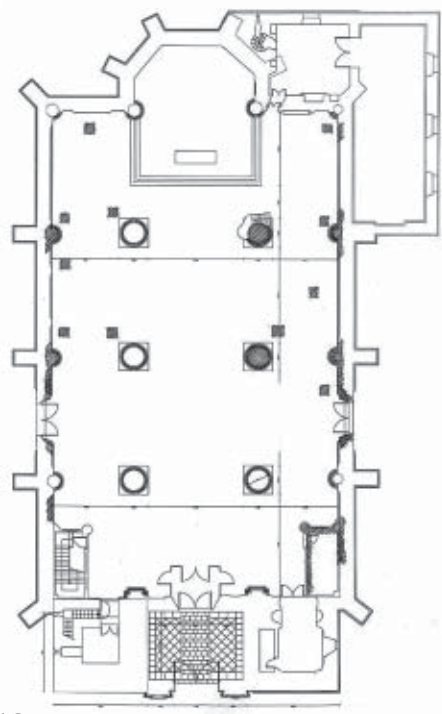

18

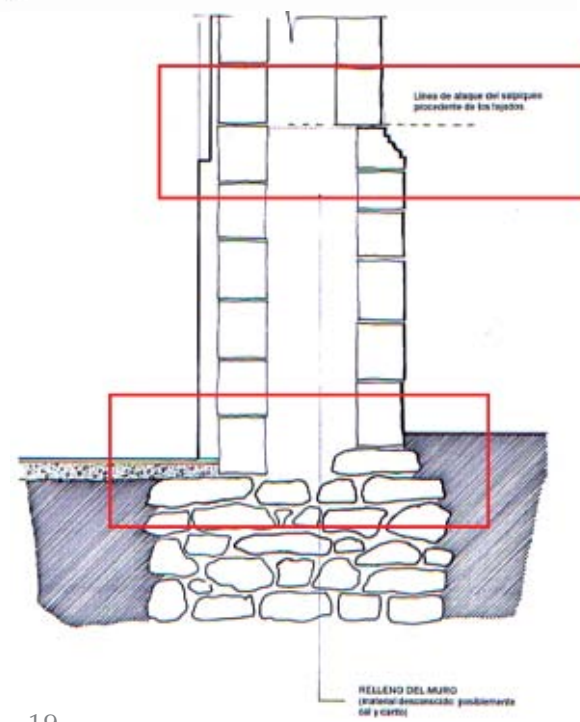

15. Catedral de Getafe. Vista general.

5. La inspección higrotérmica es útil cuando se cartografían los valores sobre planos del edificio, permitiendo correlacionar los focos con otros factores: constructivos, de riesgo de filtración, etc.

6. El Factor Intensidad de evaporación máximo en un edificio no es igual a lo largo del año (como se ha mostrado en el caso del estudio de la iglesia de San
16. Detalle del zócalo Sur en el momento de la inspección.

17. gráfico de intensidad de focos en la inspección de la catedral de Getafe.

18. Cartografía de focos detectados en la catedral de Getafe.

19. Sección del muro indicando ron filtraciones de agua. las zonas por las que se detecta- 
Pedro). En invierno, los edificios evaporan poco, por lo que se miden factores de evaporación bajos, mientras que en primavera-verano los edificios evaporan mejor, y los focos se detectan con más facilidad. En estas épocas, los edificios verdaderamente húmedos pueden Ilegar a manifestar factores de intensidad de evaporación de hasta $10 \mathrm{~g} / \mathrm{kg}$.

7. La inspección higrotérmica, por sí misma, no cuantifica el caudal de la evaporación que se produce desde muros o soleras (no habla de gramos de agua evaporados por metro cuadrado o de conceptos similares), pero es una primera aproximación al comportamiento higrotérmico global de los cerramientos del edificio.

8. El mayor inconveniente de la inspección higrotérmica es que se trata de una técnica que podríamos definir como "artesanal", lo que significa que los instrumentos actuales requieren la toma manual en cada punto de lectura. Dependiendo de la velocidad de respuesta del termohigrómetro, se pueden llegar a tomar hasta 25 lecturas por hora.

\section{BIBLIOGRAFÍA}

(1) Dunnicliff J. "Systemathic approach to planning monitoring programs using geotechnical instrumentation- an update" Geotecnical News sept 97

(2) Garcia Morales, S; Palomo, A: "Humidity studies in Quiñones Chapel, Medina del Campo, (Valladolid, Spain)". En "Structural Repair and Maintenance of Historical Buildings III,"(Actas del Congreso STREMA 93), Pág. (275- 283) (1993) Ed: C.A.Brebbia, J.Domínguez, F.Escrig. Computational Mechanics Publications. Southampton Boston

(3) Massari, G. : Risanamento igienico dei locali umidi Milano, Ulrico Hoepli Editore, 1959

(4) Rotman, P; Sanders, C; Harrison, H.: Understanding dampness . BRE, BR466, (2004), 224pp ISBN: 978-1-86081-686-4

(5) BRE Guide Assessing moisture in building materials BRE, GR33, (2002), 18pp ISBN: 978-1-86081-870-7

(6) Pardo D. "Curvas de humedad. Humedades de capilaridad" Plan Director De La Catedral De Santa Maria. Fundación Catedral Santa María. Volumen I, capítulo II, 4.1.9, pag 366. 2002

(7) Hutton, T. "Monitoring Britain’s Heritage" Construction Repair, vol 10, nº 1 (Enero-feb 96)

(8) Un avanzado sistema a base de sensores inalámbricos es el denominado "MHS", (Monitoring Heritage System), desarrollado en el seno de un Proyecto de Investigación de la Fundación Santa María la Real de Aguilar de Campoo, y todavía pendiente de publicación

(9) Sandrolini, F; Franconi, E.: "An operative protocol for reliable measurement of moisture in porous materials of ancient buildings". Building and Environment, (2005).

(10) Lerma.J. L., Cabrelles M., Portalés C. "Multitemporal thermal analysis to detect moisture on a building façade" Construction and Building Materials 25 (2011) 2190-2197

(11) Scheffler, G. A; Plagge, R. :"Application of instantaneous profile measurement of moisture content and moisture potential in porous materials". Materials and Structures, (February 2011), DOI 10.1617/s11527-011-9716-y

(12) Newman, A.J. : Improvement of the drilling method for the determination of moisture content in building materials CP22/75, Building Research Establishment, 1975

(13) Garcia Morales, S "Metodología para el diagnóstico de humedades de capilaridad y condensación higroscópica en edificios históricos" Tesis Doctoral, Universidad Politécnica de Madrid, 1995

(14) Garcia Morales, S; Palomo, A. "Moisture in Heritage Buildings. Part I. A new work Methodology" Architectural Science review Vol: 41. Pág 51-56,1998

(15) Garcia Morales, S; Palomo, A. :"Moisture in Heritage Buildings. Part II. application of the diagnostic method to the Church of San Salvador". Architectural Science review. Vol 41. Pag $57-66,1998$ 